\section{Lymphomrisiko unabhängig von Geburtsgewicht}

Fast jede zweite Krebsdiagnose bei Kindern ist eine hämatologische

Neoplasie. Bei Leukämien scheint es einen Zusammenhang mit dem Geburtsgewicht zu geben. Eine Metaanalyse ging dem auch bei Lymphomen nach.

$\mathrm{D}$ as Geburtsgewicht als leicht zu ermittelndes Maß für intrauterines Wachstum wird mit den verschiedenen hämatologischen Tumoren bei Kindern in Verbindung gebracht. Eine große Metaanalyse fand einen Zusammenhang zwischen hohem Geburtsgewicht und akuter lymphoblastischer (ALL) sowie zwischen niedrigem Gewicht und akuter myeloischer Leukämie (AML). Zu den möglichen Mechanismen gehören wachstumsfördernde Effekte von IGF (insulinlike growth factor) und das Versagen der Apoptose präleukämischer Zellen.

Die Ergebnisse epidemiologischer Studien zum Zusammenhang zwischen Geburtsgewicht und kindlichen Lymphomen sind widersprüchlich. Eine Metaanalyse berücksichtigte jetzt zwei Kohortenstudien (278.751 Kinder) und sieben FallKontroll-Studien (2.660 Fälle und 69.274 Kontrollen). Angesichts der Parallelen zur ALL ging man zumindest für das Non-
Hodgkin-Lymphom (NHL) von einer möglichen Assoziation mit einem hohen Geburtsgewicht aus.

Doch es fand sich kein statistisch signifikanter Zusammenhang zwischen hohem Geburtsgewicht ( $\geq 4 \mathrm{~kg}$ oder $\geq 3,5 \mathrm{~kg}$ je nach Studie) und dem Risiko für NHL plus Burkitt-Lymphom (Odds Ratio [OR] 1,17), Hodgkin-Lymphom (HL, OR 0,94) oder irgendein Lymphom plus BurkittLymphom (OR 1,09). Auch ein niedriges Geburtsgewicht $(<2,5 \mathrm{~kg})$ hatte keinen Effekt hinsichtlich NHL plus BurkittLymphom (OR 1,07), HL (OR 0,94) oder irgendeinem Lymphom plus BurkittLymphom (OR 1,02).

Fazit: In dieser Metaanalyse fand sich kein statistisch signifikanter Zusammenhang zwischen dem Geburtsgewicht und einem möglichen Lymphomrisiko. Möglicherweise ist das Geburtsgewicht ein zu ungenauer Indikator, um eine Verbindung

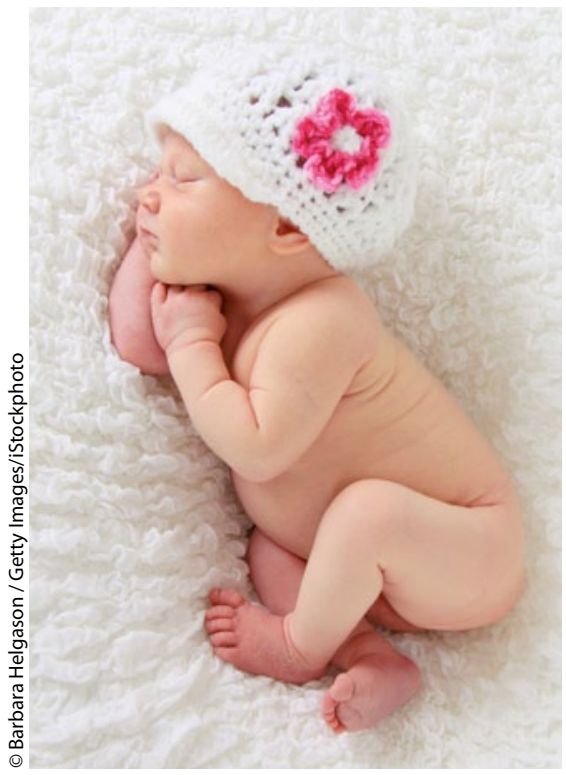

Ob es an einem Lymphom erkrankt, darüber sagt das Geburtsgewicht nicht aus.

zwischen fetalem Wachstum und Lymphomen aufzudecken.

Ulrike Wepner

Papadopoulou C et al. Is birth weight associated with childhood lymphoma? A metaanalysis. Int J Cancer. 2012;130(1):179-89.

\section{Gesund trotz Chemotherapie in Schwangerschaft}

\section{Auch schwangere Frauen unterziehen sich im Falle einer Krebserkrankung} immer häufiger einer Chemotherapie. Die Sorge über die Auswirkungen einer solchen Behandlung gilt dann insbesondere auch dem Ungeborenen.

E twa eine von 1.000-2.000 Frauen erkrankt während der Schwangerschaft an Krebs. Eine Chemotherapie wird heute üblicherweise nach dem ersten Trimenon begonnen. Über die Auswirkungen auf die Gesundheit des ungeborenen Kindes war bislang nur wenig bekannt. Die Zwischenauswertung einer multizentrischen Kohortenstudie aus Belgien, den Niederlanden und Tschechien lieferte jetzt erste Ergebnisse.

70 Neugeborene wurden median 22,3 Monate lang beobachtet und dabei neben der allgemeinen Gesundheit auf Herzfunktion und neuronale Entwicklung untersucht. Alle Kinder waren pränatal nach dem ersten Trimenon zytotoxischen Krebsmedikamenten, meist Anthrazyklinen, ausgesetzt gewesen. Die Kinder wur- den direkt nach der Geburt, mit 18 Monaten und danach im Alter von 5-6, 8-9, 11-12, 14-15 oder 18 Jahren untersucht.

Sie kamen median nach 35,7 Schwangerschaftswochen zur Welt und wogen median 2.612 g. Abgesehen von zwei frühgeborenen Zwillingen besuchten alle eine normale Schule, fast alle zeigten eine normale kognitive Entwicklung. Niedrige Scores fanden sich vorwiegend bei den Frühchen. So stieg der IQ-Score mit jedem zusätzlichen Schwangerschaftsmonat um 11,6 Punkte. Die neurologischen Untersuchungen ergaben ebensowenig Befunde außerhalb des Normbereichs wie Tests der auditorischen Funktionen oder EKG und Herzecho. Bei 39\% der Kinder über sechs Jahren ergaben sich Auffälligkeiten, wenn die Intelligenzprofile im
Einzelnen betrachtet wurden (vs. $15 \%$ in der Allgemeinbevölkerung). Diese sollten nach Ansicht der Autoren in größeren Studien weiter untersucht werden.

Fazit: Eine Chemotherapie ist auch in der Schwangerschaft ab der 14. und bis zur 35 . Woche möglich, nach den gleichen Kriterien wie bei nicht schwangeren Frauen. Die Entbindung sollte frühestens drei Wochen nach dem letzten Therapiezyklus geplant werden, wegen möglicher Auswirkungen auf die kognitive Entwicklung der Kinder sollte man Frühgeburten möglichst vermeiden. Letztlich wird die Endauswertung zeigen, wie sich eine Krebstherapie langfristig auf die ungeborenen Kinder auswirkt. Christine Starostzik

Amant $\mathrm{F}$ et al. Long-term cognitive and cardiac outcomes after prenatal exposure to chemotherapy in children aged 18 months or older: an observational study. Lancet Oncol. 2012;13(3):256-64. - Kommentar: Cardonick E. Treatment of maternal cancer and fetal development. Ibidem 218-20 